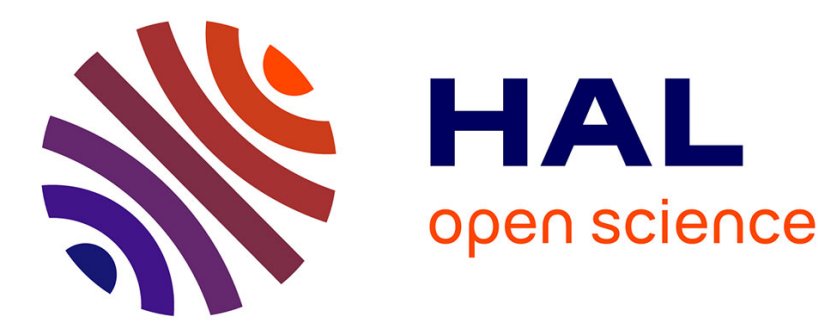

\title{
Retraction and freezing of a water film on ice
}

Virgile Thiévenaz, Christophe Josserand, Thomas Séon

\section{To cite this version:}

Virgile Thiévenaz, Christophe Josserand, Thomas Séon. Retraction and freezing of a water film on ice. Physical Review Fluids, 2020, 5 (4), 10.1103/PhysRevFluids.5.041601 . hal-02882547

\section{HAL Id: hal-02882547 https://hal.science/hal-02882547}

Submitted on 12 Nov 2020

HAL is a multi-disciplinary open access archive for the deposit and dissemination of scientific research documents, whether they are published or not. The documents may come from teaching and research institutions in France or abroad, or from public or private research centers.
L'archive ouverte pluridisciplinaire HAL, est destinée au dépôt et à la diffusion de documents scientifiques de niveau recherche, publiés ou non, émanant des établissements d'enseignement et de recherche français ou étrangers, des laboratoires publics ou privés. 


\title{
Retraction and freezing of a water film on ice
}

\author{
Virgile Thiévenaz ${ }^{1}$, Christophe Josserand ${ }^{2}$, and Thomas Séon ${ }^{1}$ \\ ${ }^{1}$ Sorbonne Universités, UPMC Univ Paris 06, UMR 7190, Institut d’Alembert, F-75005, Paris, France \\ CNRS, UMR 7190, Institut Jean Le Rond d'Alembert, F-75005, Paris, France \\ ${ }^{2}$ Laboratoire d'Hydrodynamique (LadHyX), UMR7646 CNRS-Ecole Polytechnique, IP Paris, 91128 Palaiseau CEDEX, France
}

(Dated: March 25, 2020)

\begin{abstract}
We investigate experimentally the different shapes taken by a water drop freezing during its impact on a cold surface. We show that these shapes are formed by a water film that remains on top of the first formed ice layer. The capillary hydrodynamics of this water film dewetting on its own ice, coupled with its vertical solidification, is thus quantitatively characterized, allowing us to understand and predict the formation of the emerging patterns. Finally, this experiment enables to study the contact angle dynamics, giving a deep insight into the wetting of water on ice.
\end{abstract}

When a drop impacts a solid surface, it usually highly deforms, sometimes detaches droplets or bounces, and in all cases takes splendid shapes resulting from a subtle interplay between inertia, surface tension, viscosity and substrate wetting. This iconic problem of fluid mechanics has been, and still is, extensively studied [1,2]. On the other hand, the freezing of a liquid sphere, considered in the mid- $19^{\text {th }}$ century as a model for the earth formation, probably constitutes the first solidification study of history [3, 4], and keeps intriguing researchers today [5]. This paper combines these two model problems to report on the unexpected shapes taken by frozen impacted drops, due to the interaction between the retraction of the drop after impact and its rapid freezing [6-8]. When solidification is coupled to the complex hydrodynamics of a drop impact [9], the geometry of the resulting frozen drop, called splat in metallurgy, can be very diverse, showing in particular various shapes and roughnesses [10, 11]. Knowing the shape of these splats is crucial as they enter in models of plasma spraying, a well-established means of forming thick coatings ( $300 \mu \mathrm{m})$ useful in many applications (thermal protection, resistance to corrosion, oxidation ...) [7, 12,-14]. Even without impact, the simplest case of the freezing of a sessile drop on a cold surface, already gives rise to a surprising pointy ice drop [15, 16], raising the question of the contact angle between water and ice [17]. In order to understand these complex shapes, the behavior of a water film on ice has to be clarified, in particular its retraction and wetting dynamics. Even though it has been the focus of a few studies [18, 19], water capillary dynamics on ice still remains a highly complex subject. In particular because of the disordered quasi-liquid layer on top of the surface of ice [20-23], which is affected by the surrounding gas composition [24], and explains ice slipperiness [25].

In this Letter, we investigate the retraction of liquid water on ice which occurs after a drop impacts a cold subfreezing surface. We show for the first time how the capillary hydrodynamics of a water film coupled with its solidification can build unexpected patterns (Fig. 11). Thus, we explore a new way of studying the wetting of water on ice and of estimating the ice-water contact angle.

The drop impact setup consists of releasing a water drop at room temperature from a capillary tube using a syringe pump [26]. We consider two drop radii: $R_{0}=1.9 \mathrm{~mm}$ and $1.2 \mathrm{~mm}$. The impact velocity $U_{0}$, controlled by the height of fall, ranges from $1.7 \mathrm{~m} . \mathrm{s}^{-1}$ to $3 \mathrm{~m} . \mathrm{s}^{-1}$. We use three large blocks $(100 \times 100 \times 30 \mathrm{~mm})$ of different materials (steel, copper, marble) as substrates, their distinct thermal properties [27] allowing us to change the rate of freezing [26, 28, 29]. They are cooled down between 0 and $-80{ }^{\circ} \mathrm{C}$ using liquid nitrogen. This experiment is placed inside a dry air chamber in order to minimize the frost formation. The impact dynamic is studied using a high-speed camera, and the height profile of the frozen drop is extracted with a polychromatic confocal sensor moving along a translation platform [27].

The impact process is represented by two timelines (Fig. 11(a)\&(b)) corresponding to two different substrate temperatures, $-10^{\circ} \mathrm{C}$ and $-30{ }^{\circ} \mathrm{C}$ (see corresponding videos in [27]). In the first milliseconds of the impact the drop spreads and reaches its maximal diameter while a thin layer of ice freezes beneath it. Before the third image of each sequence, the system is in the configuration described by Fig. 1.c): the liquid film is pinned at the edge of a thin ice layer. Freezing goes on and the ice layer thickness follows the classical self-similar law $h(t)=\sqrt{D_{\text {eff }} t}$ given by solving the Stefan problem [4]. This latter consider a solidification front propagating between two phases (liquid and solid) of the same material, without substrate. $D_{\text {eff }}$ is the effective diffusion coefficient of the propagation dynamics of the ice-water front solidification. As shown in a previous article [26] and used here, an excellent estimation of the effective diffusion coefficient $D_{\text {eff }}$ may be obtained by taking into account the heat propagation in the substrate. At the fourth image, the contact angle has relaxed down to its deweting value (Fig. 11.d) and the thickness of the ice layer at this point is called $h_{p}$.

Between the fourth and the sixth image the liquid retracts on ice, leading to the two different shapes: a spherical cap (Fig. 1](a)) or a half-ring (Fig. 11 (b)) of water on top of a thin ice disc. Finally, the remaining liquid finishes freezing 


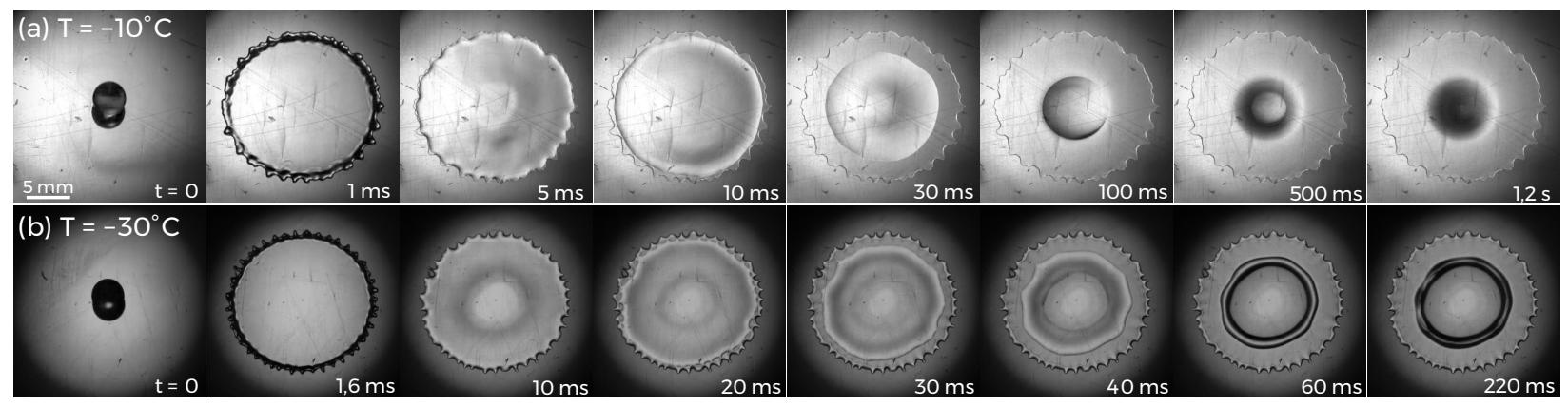

(c)

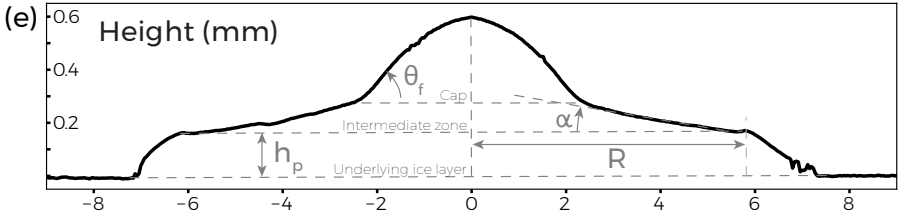

(d)
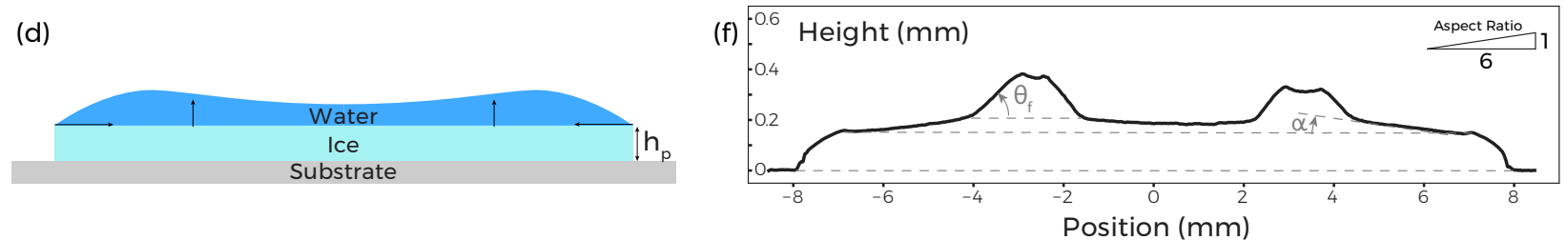

FIG. 1. (a)\&(b) Images sequences of the impact and retraction of a water drop on a cold aluminium surface at two different temperatures. The liquid film starts retracting on ice about $10 \mathrm{~ms}$ after the impact. Depending on the substrate temperature (a: $-10{ }^{\circ} \mathrm{C}, \mathrm{b}:-30{ }^{\circ} \mathrm{C}$ ), the film will or will not have the time to fully retract, yielding different patterns (see corresponding videos in [27]). (c)\&(d) Schematic view of the first instants of a drop impacting a supercooled substrate, in particular the ice and water dynamics. (e)\&(f) Height profiles extracted from experiments similar to (a)\&(b). The two ice structures will be referred as cap (a,e) and ring patterns $(\mathrm{b}, \mathrm{f})$. All the geometrical quantities used in this paper are defined here. The uncertainty $\Delta h$ is of order $1 \mu \mathrm{m}$.

quasi-statically, over a few hundreds of milliseconds. The retraction of a liquid on its own solid phase is not $a$ priori expected thermodynamically, this is in fact not observed for molten metal or wax drop impacts [30, 31] and seems to be a peculiar feature of water. It indicates in particular that liquid water and ice exhibit a non-zero contact angle as it has already been observed in a few other configurations [17, 32, 34].

As a consequence of this competition between capillary retraction and solidification, the different shapes adopted by the frozen drop depend on the impact parameters and the substrate temperature. For a constant set of impact parameters, when the substrate temperature is slightly below the melting temperature, the frozen structure takes the shape of the spherical cap on top of a thin disc, whereas when the substrate temperature is colder, the final shape is the half-ring. Figures 1 (e)\&(f) present the height profiles corresponding to the two shapes and define the notations used in the following. Note that the aspect ratio of these two figures is 6 , meaning that these ice structures are really flat: typically a few hundreds of microns thick and about one centimeter wide. On both profiles we can observe three different zones: the underlying ice layer of thickness $h_{p}$, the pattern on the top, which can be a spherical cap (Fig 1 e) or a ring (Fig 1f), and an intermediate zone. We define the angle $\alpha$ as the angle between the ice-air interface and the horizontal in the intermediate zone, and the angle $\theta_{\mathrm{f}}$ between the pattern and the horizontal. In the following, our goal is to quantitatively characterize the formation of such ice structures from the generic configuration of Fig. 1 (d) and, in particular, to discuss the final angle $\theta_{\mathrm{f}}$ on the solid structure.

Let us first consider the retraction of the water film on the ice disc. Fig. 2(a) presents the film radius versus time showing that the retraction velocity $V_{\text {ret }}$ is constant during most of the retraction. Subsequently, the retraction velocity is plotted in Fig. 2(b) for each experiment versus $\Delta T=T_{m}-T_{s}$, with $T_{m}$ the melting temperature $-0{ }^{\circ} \mathrm{C}$ in our case - and $T_{s}$ the substrate temperature. We observe that $V_{\text {ret }}$ is roughly the same for any value of the control parameters, represented by different markers: it does not vary with impact velocity, drop radius, substrate material and temperature. Hence, we deduce the retraction velocity of water on ice:

$$
V_{\text {ret }} \simeq 17 \pm 3 \mathrm{~mm} \cdot \mathrm{s}^{-1}
$$

Yet, those control parameters do have an effect on the thickness of the liquid film which retracts on ice, which means 

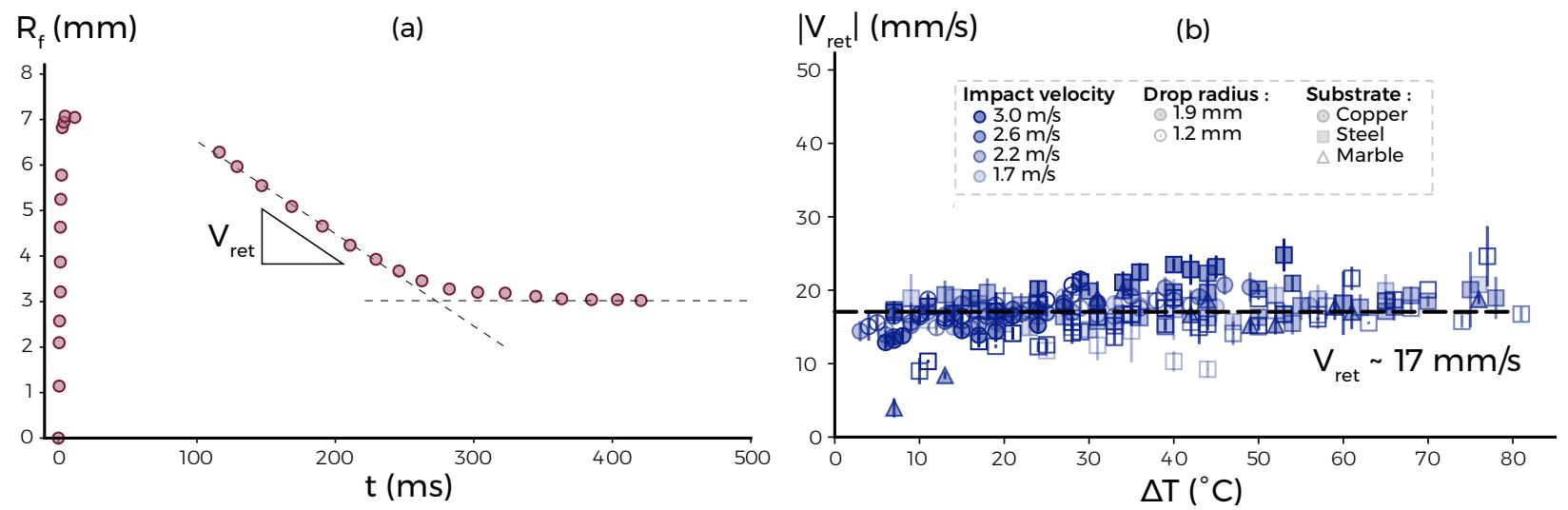

FIG. 2. (a) Time evolution of the liquid film radius $R_{\mathrm{f}}$ for a single experiment. $R_{\mathrm{f}}$ decreases linearly during the retraction, therefore pointing at a constant retraction velocity $V_{\text {ret }}$. (b) Retraction velocity of the water film on ice, as a function of $\Delta T=T_{m}-T_{s}$, for a large set of experiments with a Weber number ranging from 48 to 234. The top inset shows the meaning of the different markers: impact velocities are represented by different colours (from dark blue for the fastest to light blue for the slowest), substrates by symbols (circles for copper, squares for steel and triangles for marble) and drop radii by the filling (full symbols for $R_{0}=1.9 \mathrm{~mm}$, empty symbols for $R_{0}=1.2 \mathrm{~mm}$. The retraction velocity does not vary with the control parameters nor with the film thickness.

that $V_{\text {ret }}$ does not depend on the film thickness. As the film thickness appears in the Taylor-Culick velocity [35] $\left(\left(\sqrt{\gamma /\left(\rho h_{f}\right)}\right)\right.$, this observation suggests that the capillary retraction is here balanced by viscosity, rather than by inertia.

In our experiment the ice keeps growing as long as there is water on it, so that the liquid film freezes as it retracts. The interplay between capillary retraction and solidification therefore controls the final shape of the frozen drop, and may be characterized by comparing the dynamics of both processes, which we do through scaling laws. Knowing the growth dynamics of ice $\left(h(t)=\sqrt{D_{\text {eff }} t}\right)$, we can determine the solidification velocity $V_{\text {sol }}\left(d h / d t=\sqrt{D_{\text {eff }}} /(2 \sqrt{t})\right)$ at the moment water starts retracting $\left(t=h_{\mathrm{p}}^{2} / D_{\text {eff }}\right)$ :

$$
V_{\text {sol }}=\frac{D_{\text {eff }}}{2 h_{\mathrm{p}}}
$$

Fig. 3 a) shows the variation of $\tan (\alpha)$ (see Fig. 1 (e)\&(f)) versus the ratio of the solidification and retraction velocities, for all our experiments. All the data gather along a line $\tan \alpha \simeq 0.67 V_{\text {sol }} / V_{\text {ret }}$, which demonstrates that the ice slope $\alpha$ is indeed the result of the balance between vertical solidification and radial retraction. The lesser-than-one 0.67 factor is probably due to an overestimation of $V_{\text {sol }}$, as it is defined at the beginning of the retraction whereas the real instant velocity decreases over time.

Now, we question the mechanism that select the final pattern: cap (Fig. 1(e)) or ring (Fig. 1(f)). We observe on the timelines (Fig. 1 ( a)\&(b)) that during the relaxation of the contact line (between the second and the fourth image, schematized by the transition from Fig. 1 (c) to (d)), a rim appears at the edge of the liquid film creating a trough at the center [36, 37]. When this film with a curved free surface retracts two options exist: either the freezing rate is slow compared to the retraction and the rim will eventually collapse into a cap shape (Fig. 11(a)\&(e)), either the freezing is quick enough to reach the trough before the rim collapses, in which case a liquid ring is left to freeze, yielding the ring shape (Fig. [1](b)\&(f)). In order to study the transition from one shape to another, the timescales of retraction $\tau_{\text {ret }}$ and solidification $\tau_{\text {sol }}$ can be estimated using the characteristic lengths $R$ and $h_{\mathrm{f}}$, respectively the liquid film radius and thickness at the onset of retraction, measured from the thickness profiles (see more details in the [27]), yielding:

$$
\tau_{\text {ret }}=\frac{R}{V_{\text {ret }}} \quad \text { and } \quad \tau_{\text {sol }}=\frac{h_{\mathrm{f}}}{V_{\text {sol }}}=\frac{2 h_{\mathrm{f}} h_{\mathrm{p}}}{D_{\text {eff }}}
$$

Figure 3 b) is a phase diagram that plots the timescales $\tau_{\text {ret }}$ versus $\tau_{\text {sol }}$ for our large range of control parameters values (see legend on Fig. 2(b)), by distinguishing the cap (in red) and ring (in blue) experiments We obtain a clear separation between the two shapes, shown with a dashed line, validating the proposed mechanism of pattern selection. The dashed line has a slope of 3, larger than 1, which may be explained through two distinct contributions: the use of the film radius $R$ that overestimates $\tau_{\text {ret }}$ since the film only retracts on a fraction of it, and the previously described overestimation of $V_{\text {sol }}$ which translates into an underestimation of $\tau_{\text {sol }}$. 
(a)

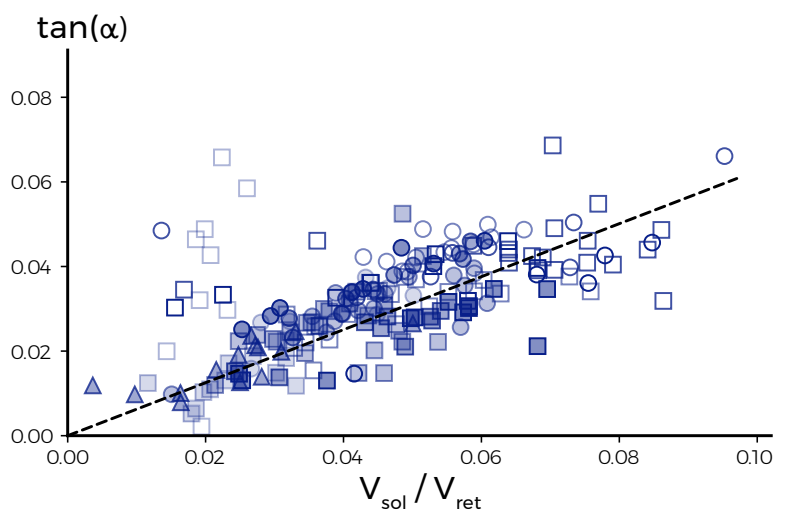

(b)

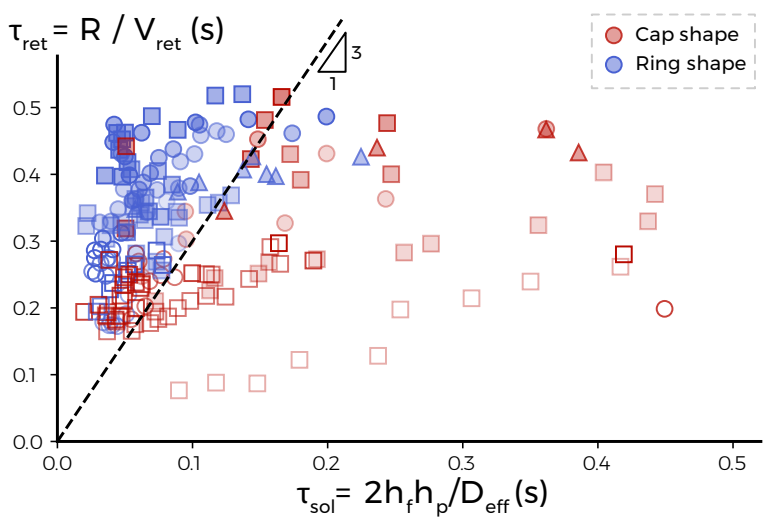

FIG. 3. Interactions between retraction and solidification. (a) Tangent of angle $\alpha$ as defined on the figure 1 (e)\&(f), versus of the ratio of the solidification and the retraction velocities $V_{\text {sol }} / V_{\text {ret. }}$. The set of experiments is the same as in figure 2b) and the legend is also identical. The dashed line is the best linear fit with a slope 0.67. (b) Phase diagram of the possible final shapes, cap (red, see Fig. 1 (a)) and ring (blue, see Fig. 1](b)), depending on the characteristic times of solidification $\tau_{\text {sol }}$ and retraction $\tau_{\text {ret. }}$. The symbols shape and opacity represents the impact and freezing parameters, according to figure 2 b) legend. The dashed line has a slope of 3 and is to guide eye.

The main exception to our criterion concerns the group of empty red markers in the lower left part of Figure 3 (b), which represents a series of impacts with small drops yielding a cap shape (red) whereas they should yield a ring shape (blue). This mismatch can be understood as the effect of the small width of the retracting liquid film which results from the impact of smaller drops: if the film is not spread enough no rim will form, and therefore no ice ring will freeze whatever the solidification time. However, although this explanation qualitatively explains our data, we have unfortunately not been able to find a simple criterion quantifying the minimal size required to form a retraction rim.

Either way, the scaling analyses presented in figure 3 prove that the final shape of the frozen drop, be it the angle $\alpha$ or the pattern, ring or cap, is entirely defined by the competition between retraction and solidification. Note that another shape with two concentric rings instead of one can been observed, but this configuration is the result of the same mechanism that forms one ring.

Finally, this experiment provides an original way to progress on the wetting of water on ice. Indeed, the angle $\theta_{\mathrm{f}}$ (Fig. 1 (e)\&(f)) is related to the contact angle that water made with ice at the moment it has been frozen, even if it probably only gives an indirect measurement of the real contact angle. Indeed, the density variation during solidification might change this angle. Moreover, we may wonder whether the solid-liquid interface is horizontal during the solidification, although it seems supported by the simple relation between $\tan \alpha, V_{\text {ret }}$ and $V_{\text {sol }}$ (Fig. 3 (a)).

Figure 4 presents the variation of $\theta_{\mathrm{f}}$ for all our experiments, versus the ratio of the characteristic times of solidification $\tau_{\text {sol }}$ and retraction $\tau_{\text {ret }}$. We first observe a whole range of $\theta_{\mathrm{f}}$ from less than one degree up to about fifteen degrees in the ring configuration $\left(\tau_{\text {ret }}>3 \tau_{\text {sol }}\right)$, when the film is solidified while it is retracting. Note that, as the retraction velocity is constant (Fig. 2), we would expect a constant value of the corresponding retraction angle that we do not observe on this graph. Indeed, in this case the receding contact line is frozen by the solidification front before water reached an equilibrium shape. Consequently, one should expect a strong modification of the water-ice angle due to capillary relaxation of the remaining liquid being faster than the solidification front propagation. This relaxation dynamics while freezing explains the difference between $\theta_{\mathrm{f}}$ and the real contact angle, and this difference should depend on the experimental parameters and is difficult to predict. However, when the water film has time to form an equilibrium spherical cap before being frozen $\left(\tau_{\text {ret }}<3 \tau_{\text {sol }}\right)$, its contact angle with the ice always reaches a constant limit value, for any control parameters. Depending whether a contact angle hysteresis exists or not [38], this particular angle may be an equilibrium angle or a retraction angle, but in either case it exists, is unique and constant. Its value when solidified is called the solidified equilibrium angle $\theta_{\mathrm{eq}}^{\text {sol }}$.

Despite the difference between the real contact angle and $\theta_{\mathrm{f}}$, it is interesting to compare our value $\theta_{\mathrm{eq}}^{\text {sol }} \sim 12^{\circ}$ with those reported previously in the literature. In fact, to our knowledge, only few studies have studied the ice-water contact angle and their results span a large range from $1^{\circ}$ up to $40^{\circ}[18,19,32,-34,39,40]$. Knight [32] notably measured $12^{\circ}$ in 1966 for the receding contact angle of water on ice by observing the retraction of a freezing puddle. On the other hand, as the interfacial tension between ice and water is very low due to their high affinity [34], Young's relation imposes that a non-zero contact angle of water on ice is equivalent to a surface free energy of ice lower than the 


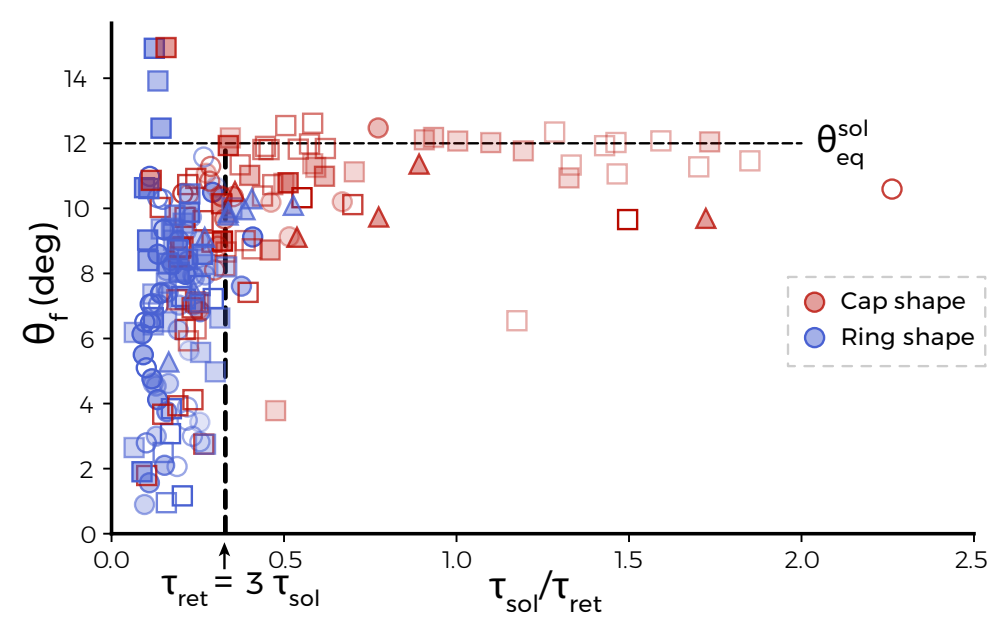

FIG. 4. Final contact angle $\theta_{\mathrm{f}}$ of the retracting water film on ice versus the ratio of the characteristic times of solidification and retraction: $\tau_{\mathrm{sol}} / \tau_{\text {ret }}$. For low values of this time ratio, $\theta_{\mathrm{f}}$ varie between $1^{\circ}$ and $12^{\circ}$. For high values of this time ratio, $\theta_{\mathrm{f}}$ reaches a constant value $\theta_{\mathrm{f}}=12^{\circ} \pm 1^{\circ}$. The vertical dashed line shows threshold that delimited the two ice shapes: $\tau_{\text {ret }} / \tau_{\text {sol }}=3$, as in figure 3 (b). The symbols shape and opacity represents the impact and freezing parameters, according to Fig. 2 b) legend.

surface tension of water at $0{ }^{\circ} \mathrm{C}, 75.6 \mathrm{~mJ} / \mathrm{m}^{2}$ [41]. Van Oss et al. [34] found accordingly that the surface free energy of ice is $69.2 \mathrm{~mJ} / \mathrm{m}^{2}$, which puts the contact angle of water on ice around $24^{\circ}$. Our value of $\theta_{\mathrm{eq}}^{\text {sol }}$ is therefore consistent with this range of value, but still does not help to discriminate between the preceding observations. However, if the relation between our solid equilibrium contact angle $\theta_{\mathrm{eq}}^{\text {sol }}$ and the real ice-water contact angle can be estimated by further research, this experiment would provide a new value of the contact angle of water on ice.

As a conclusion, the final shape of the frozen drop is determined by the competition between the dynamics of retraction and freezing. Moreover, our experiments enable to characterize the retraction of water on ice, in terms of retraction velocity and wetting. We showed in particular that the water film retracts at a constant velocity which does not depend on the temperature nor on the film thickness. This study also outlines the contact angle dynamics during the retraction of water on ice and provides an alternative set-up to characterize the equilibrium contact angle in the future. Further investigations into this wetting dynamic, especially its possible link to surface melting of ice [33], might shed more light on the nature of the surface of ice [42].

[1] A. M. Worthington. On the forms assumed by drops of liquids falling vertically on a horizontal plate. Proceedings of the Royal Society of London, 25:261-272, 1876 - 1877.

[2] C Josserand and ST Thoroddsen. Drop impact on a solid surface. Annual Review of Fluid Mechanics, 48:365-391, 2016.

[3] G Lamé and BP Clapeyron. Mémoire sur la solidification par refroidissement d'un globe liquide. In Annales Chimie Physique, volume 47, pages 250-256, 1831.

[4] L.I. Rubinstein. The Stefan Problem, volume 27 of Translations of Mathematical Monographs. American Mathematical Soc., 1971.

[5] Sander Wildeman, Sebastian Sterl, Chao Sun, and Detlef Lohse. Fast dynamics of water droplets freezing from the outside in. Physical Review Letters, 118(8):084101, 2017.

[6] Shiraz D Aziz and Sanjeev Chandra. Impact, recoil and splashing of molten metal droplets. International journal of heat and mass transfer, 43(16):2841-2857, 2000.

[7] Rajeev Dhiman, André G McDonald, and Sanjeev Chandra. Predicting splat morphology in a thermal spray process. Surface and Coatings Technology, 201(18):7789-7801, 2007.

[8] Elisabeth Ghabache, Christophe Josserand, and Thomas Séon. Frozen impacted drop: From fragmentation to hierarchical crack patterns. Physical Review Letters, 117(7):074501, 2016.

[9] Ilia V Roisman. Fast forced liquid film spreading on a substrate: flow, heat transfer and phase transition. Journal of Fluid Mechanics, 656:189-204, 2010.

[10] Rajeev Dhiman and Sanjeev Chandra. Freezing-induced splashing during impact of molten metal droplets with high weber numbers. International journal of heat and mass transfer, 48(25):5625-5638, 2005.

[11] Sanjeev Chandra and Pierre Fauchais. Formation of solid splats during thermal spray deposition. Journal of Thermal Spray Technology, 18(2):148-180, 2009. 
[12] J Madejski. Solidification of droplets on a cold surface. International Journal of Heat and Mass Transfer, 19(9):1009-1013, 1976.

[13] R Bhola and S Chandra. Parameters controlling solidification of molten wax droplets falling on a solid surface. Journal of materials science, 34(19):4883-4894, 1999.

[14] P Fauchais, A Vardelle, M Vardelle, and M Fukumoto. Knowledge concerning splat formation: an invited review. Journal of Thermal Spray Technology, 13(3):337-360, 2004.

[15] WW Schultz, MG Worster, and DM Anderson. Solidifying sessile water droplets. In Interactive dynamics of convection and solidification, pages 209-226. Springer, 2001.

[16] Alvaro G Marin, Oscar R Enriquez, Philippe Brunet, Pierre Colinet, and Jacco H Snoeijer. Universality of tip singularity formation in freezing water drops. Physical review letters, 113(5):054301, 2014.

[17] DM Anderson, M Grae Worster, and SH Davis. The case for a dynamic contact angle in containerless solidification. Journal of crystal growth, 163(3):329-338, 1996.

[18] Charles A Knight. Experiments on the contact angle of water on ice. Philosophical magazine, 23(181):153-165, 1971.

[19] Victor F Petrenko and Robert W Whitworth. Physics of ice. Clarendon Press, 1999.

[20] Joost WM Frenken and JF Van der Veen. Observation of surface melting. Physical review letters, 54(2):134, 1985.

[21] A. Lied, H. Dosch, and J.H. Bilgram. Surface melting of lce lh single crystals revealed by glancing angle x-ray scattering. Phys. Rev. Lett., 72(22):3554, 1994.

[22] Yimin Li and Gabor A Somorjai. Surface premelting of ice. The Journal of Physical Chemistry C, 111(27):9631-9637, 2007.

[23] Gen Sazaki, Salvador Zepeda, Shunichi Nakatsubo, Makoto Yokomine, and Yoshinori Furukawa. Quasi-liquid layers on ice crystal surfaces are made up of two different phases. Proceedings of the National Academy of Sciences, 109(4):1052-1055, 2012.

[24] Michael Elbaum, SG Lipson, and JG Dash. Optical study of surface melting on ice. Journal of crystal growth, 129(3-4):491505, 1993.

[25] Robert Rosenberg. Why is ice slippery? Physics Today, 58(12):50, 2005.

[26] Virgile Thiévenaz, Thomas Séon, and Christophe Josserand. Solidification dynamics of an impacted drop. Journal of Fluid Mechanics, 874:756-773, 2019.

[27] See supplementary material at [url] for details on the experimental set-up and material properties, as well as videos of the two impact regime.

[28] T Bennett and D Poulikakos. Heat transfer aspects of splat-quench solidification: modelling and experiment. Journal of Materials Science, 29(8):2025-2039, 1994.

[29] Jolet de Ruiter, Dan Soto, and Kripa K Varanasi. Self-peeling of impacting droplets. Nature Physics, 14(1):35, 2018.

[30] Stefano Schiaffino and Ain A Sonin. Motion and arrest of a molten contact line on a cold surface: an experimental study. Physics of Fluids (1994-present), 9(8):2217-2226, 1997.

[31] S. Schiaffino and A.A. Sonin. Molten droplet deposition and solidification at low weber numbers. Phys. Fluids, 9:3172, 1997.

[32] Charles A Knight. The contact angle of water on ice. Journal of Colloid and Interface Science, 25(2):280-284, 1966.

[33] Lasse Makkonen. Surface melting of ice. The Journal of Physical Chemistry B, 101(32):6196-6200, 1997.

[34] CJ Van Oss, RF Giese, R Wentzek, J Norris, and EM Chuvilin. Surface tension parameters of ice obtained from contact angle data and from positive and negative particle adhesion to advancing freezing fronts. Journal of adhesion science and technology, 6(4):503-516, 1992.

[35] F.E.C. Culick. Comments on a ruptured soap film. J. Appl. Phys., 31:1128, 1960.

[36] Marco Rivetti, Thomas Salez, Michael Benzaquen, Elie Raphaël, and Oliver Bäumchen. Universal contact-line dynamics at the nanoscale. Soft Matter, 11(48):9247-9253, 2015.

[37] Andrew MJ Edwards, Rodrigo Ledesma-Aguilar, Michael I Newton, Carl V Brown, and Glen McHale. Not spreading in reverse: The dewetting of a liquid film into a single drop. Science Advances, 2(9):e1600183, 2016.

[38] D. Bonn, J. Eggers, J. Indekeu, J. Meunier, and E. Rolley. Wetting and spreading. Rev. Mod. Phys., 81:739, 2009.

[39] WM Ketcham and PV Hobbs. An experimental determination of the surface energies of ice. Philosophical Magazine, 19(162):1161-1173, 1969.

[40] Jaroslaw Drelich, Emil Chibowski, Dennis Desheng Meng, and Konrad Terpilowski. Hydrophilic and superhydrophilic surfaces and materials. Soft Matter, 7(21):9804-9828, 2011.

[41] David R Lide. CRC handbook of chemistry and physics. CRC Press, Boca Raton, FL, 2005.

[42] Yuki Nagata, Tetsuya Hama, Ellen HG Backus, Markus Mezger, Daniel Bonn, Mischa Bonn, and Gen Sazaki. The surface of ice under equilibrium and nonequilibrium conditions. Accounts of chemical research, 2019. 